\title{
Random Phase Updating Algorithm for OFDM Transmission With Low PAPR
}

\author{
Homayoun Nikookar and Knut Sverre Lidsheim
}

\begin{abstract}
A novel random phase updating algorithm for the peak-to-average power ratio (PAPR) reduction of the OFDM signal is addressed. The phase of each subcarrier is updated by a random increment until the PAPR goes below a certain threshold level. The influence of different distributions for the phase increments and the variance of distributions on the mean and variance of PAPR as well as the number of iterations to reach the threshold, is investigated. Further, the random phase updating algorithm has been extended by dynamically reducing the threshold level. In this method after successful updating of the phase shifts the threshold level is reduced and the variance of the phase increments is changed. Simulation results of the algorithm are provided. It is shown that the random phase updating algorithm with dynamic threshold gives the best results and can reduce the mean power variance of an 8-carrier OFDM signal with BPSK modulation, by a factor of $7 \mathrm{~dB}$. In order to reduce the complexity, the random phase updating algorithm is investigated with quantization and grouping of the phase shifts. Results show that for a 16-carrier OFDM system, 2-level quantization of phase shifts in 8 groups of 2 carriers give no significant increase in the power variance while reducing complexity. Further in the paper, the impact of phasing on the bit error rate performance of the OFDM system is studied.
\end{abstract}

Index Terms-Bit error rate, dynamic threshold, OFDM, Peak-to-Average Power Ratio (PAPR), phase updating algorithm, power variance.

\section{INTRODUCTION}

$\mathbf{O}$ FDM is the basis technology for a number of communication systems such as Digital Audio Broadcasting (DAB), Digital Video Broadcasting (DVB), Hiperlan-2, IEEE 802.11 and Digital Subscriber Lines (xDSL). In the OFDM transmission the spectra of subchannels overlap while satisfying orthogonality, giving rise to spectral efficiency. In wireless communications by using OFDM method the effect of fading is spread over many bits. Therefore, instead of few adjacent bits to be completely destroyed by the fading, each bit is slightly affected by the fading. In OFDM method because of parallel transmission the symbol period is increased. This has the added advantage of this technique to work in the channels having impulsive noise characteristics. Other advantage of the OFDM technique is its implementation with the fast Fourier transform (FFT) algorithm which provides full digital implementation of the modulator and demodulator. Despite the promising properties of OFDM, its major drawback is the high PAPR which is not favorable for the power amplifiers that work in the nonlinear region. The summing of the orthogonal frequencies

Manuscript received October 23, 2001; revised March 19, 2002.

The authors are with the International Research Center for Telecommunications-Transmission and Radar (IRCTR), Faculty of Information Technology and Systems, Delft University of Technology, 2600 GA Delft, The Netherlands (e-mail: H.Nikookar@IRCTR.TUDelft.NL).

Publisher Item Identifier S 0018-9316(02)05255-1.

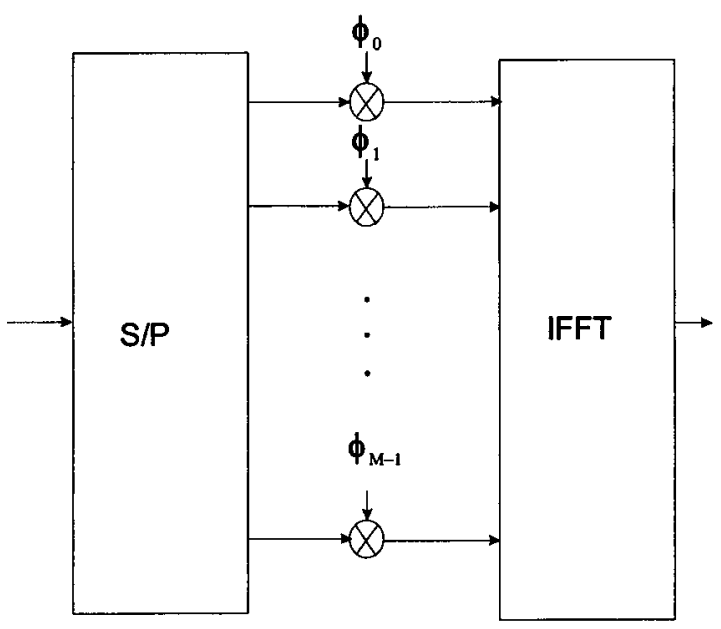

Fig. 1. Block diagram of OFDM with phasing showing the principle of adding phase shifts to the OFDM symbols.

in the FFT causes the high PAPR of the OFDM signal. When more subcarriers are in phase this results in a high PAPR.

Different methods have been proposed to mitigate the PAPR problem of OFDM. These techniques are mainly divided into two categories: Signal scrambling and Signal distortion techniques. Signal scrambling techniques are all variations on how to modify the phases to decrease the PAPR. Signal scrambling methods are reported in [1]-[5]. Some of the signal scrambling algorithms give a very low PAPR but are often difficult to be realized in practice. More practical solutions are block coding [1], selected mapping [2] and partial transmit sequences [4]. In [6] the tone reservation method is suggested to reduce PAPR. In this method a fraction of bandwidth is used to synthesize signals that are of opposite polarity and shape a peak in the OFDM signal. Subtraction of peaks reduces the PAPR without great effect on the transmission capability of OFDM. The signal distortion techniques are mainly developed to reduce the high peaks directly by distorting the signal prior to amplification. The easiest and most obvious signal distortion technique is the envelope clipping [7]. However, clipping introduces both in-band and out-of-band radiation. Other signal distortion techniques with better spectral properties are peak windowing [8], peak cancellation [9], peak power suppression [10], weighted multicarrier transmission [11], companding [12] and predistortion [13].

This paper addresses the PAPR reduction of OFDM by random phase shift updating. The paper is organized as follows: In Section II the PAPR of OFDM signal is explained. PAPR reduction of the OFDM signal by the random phase updating method is discussed in Section III and different phase distributions are investigated added by the simulation results. In Section IV, the random phase updating algorithm with dynamic 


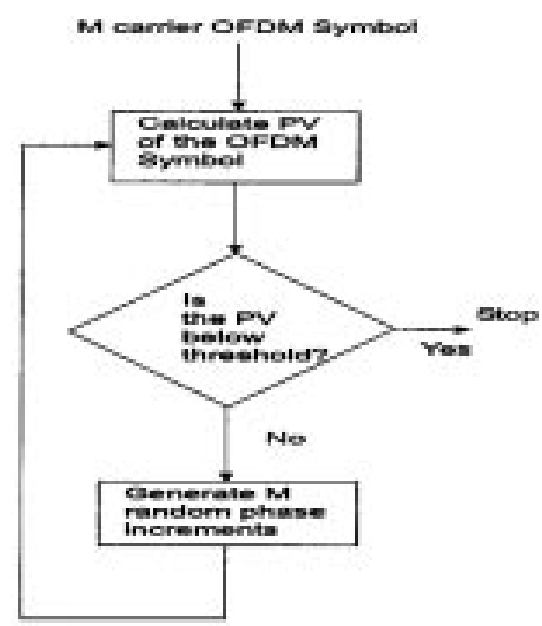

(A)

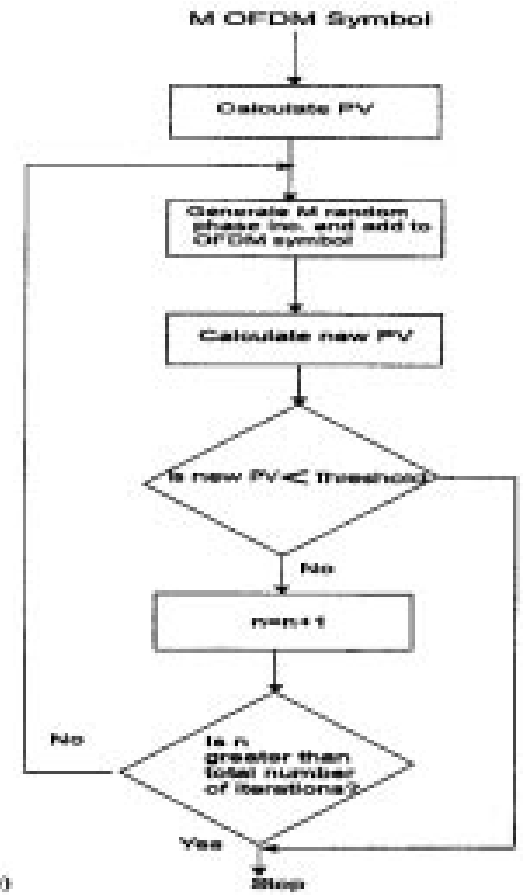

Fig. 2. Flowchart showing the iterative random phase updating algorithm, (A) with threshold, (B) with limited number of iterations.

threshold is proposed and its simulation results are depicted. The impact of random phasing on the bit error rate (BER) performance of OFDM system is investigated in Section V. Concluding remarks appear in Section VI.

\section{PAPR OF OFDM SIGNAL}

The OFDM signal can be written as

$$
s(t)=\sum_{i=-\infty}^{\infty} \sum_{m=0}^{M-1} b_{m}(i) e^{j 2 \pi(m / T)(t-i T)} p(t-i T)
$$

where $T$ is the OFDM symbol duration, $b_{m}(i)$ is the symbol of the $m$ th subchannel at time interval $i T$, which is \pm 1 for BPSK modulation, $p(t)$ is a rectangular function with amplitude one and duration $T$, and $M$ is the number of carriers. The OFDM signal of (1) in the time interval of $0 \leq t \leq T$ can be written as

$$
s(t)=\sum_{m=0}^{M-1} b_{m} e^{j 2 \pi(m / T) t} .
$$

The power of $s(t)$ is

$$
P(t)=|s(t)|^{2}=\sum_{m=0}^{M-1} \sum_{n=0}^{M-1} b_{m} b_{n}^{*} e^{j(2 \pi(m-n) / T) t} .
$$

The PAPR of the OFDM signal is written as

$$
P A P R=\frac{\operatorname{Max}\{P(t)\}}{\operatorname{Mean}\{P(t)\}} .
$$

The variation of the instantaneous power of OFDM signal from the average is $\Delta P(t)=P(t)-E[P(t)]$, and accordingly, the
TABLE I

POWER VARIANCE AND NUMBer OF ITERATIONS FOR THE RANDOM PHASE UPDATING ALGORITHM WITH UNIFORM AND GAUSSIAN DISTRIBUTIONS OF PHASE INCREMENTS

\begin{tabular}{|c|c|c|c|c|c|c|c|c|}
\hline & \multicolumn{4}{|c|}{ Uniform distribution } & \multicolumn{4}{c|}{ Normal distribution } \\
\hline & \multicolumn{2}{|c|}{$\begin{array}{c}\text { Power } \\
\text { variance }\end{array}$} & \multicolumn{2}{c|}{$\begin{array}{c}\text { No. of } \\
\text { iterations }\end{array}$} & \multicolumn{2}{c|}{$\begin{array}{c}\text { Power } \\
\text { variance }\end{array}$} & \multicolumn{2}{c|}{$\begin{array}{c}\text { No. of } \\
\text { iterations }\end{array}$} \\
\hline $\mathrm{X}$ & Mean & Std. dev. & Mean & Std. dev. & Mean & Std. dev. & Mean & Std. dev. \\
\hline 0.1 & 71.89 & 9.02 & 33.38 & 42.5 & 72.12 & 8.97 & 39.05 & 50 \\
\hline 0.25 & 68.85 & 9.59 & 58.56 & 10.6 & 69.08 & 9.57 & 9.54 & 11.8 \\
\hline 0.5 & 67.57 & 9.99 & 4.93 & 6.16 & 67.64 & 9.92 & 5.15 & 6.39 \\
\hline 0.75 & 67.47 & 10 & 4.62 & 5.75 & 67.45 & 10 & 4.67 & 5.81 \\
\hline 1.0 & 67.41 & 10 & 4.64 & 5.8 & 67.36 & 10 & 4.64 & 5.76 \\
\hline
\end{tabular}

power variance (PV) of OFDM signal, denoted by $\rho$, can be written as [3]:

$$
\rho=\frac{1}{T} \int_{0}^{T}(\Delta P(t))^{2} d t=\sum_{i=1}^{M-1}\left|R_{b b}(i)\right|^{2}
$$

where $R_{b b}(i)$ is the autocorrelation function of the sequence $b_{m}$.

$$
R_{b b}(i)=\sum_{m=0}^{M-1-i} b_{m} b_{m+i}^{*}
$$

The power variance $\rho$ is a good measure of the PAPR. PV and PAPR are related to each other according to the following relationship [11]:

$$
Q\left(\frac{P A P R-1}{\sqrt{\rho}}\right)+Q\left(\frac{1}{\sqrt{\rho}}\right)=\beta
$$

where $\beta$ denotes the probability that $P(t)$ be less than or equal to $P_{\max }$ and

$$
Q(y)=\frac{1}{\sqrt{2 \pi}} \int_{y}^{\infty} e^{-u^{2} / 2} d u
$$


From (7) it is seen that for a fixed $\beta$ the OFDM signal with high PAPR has a high value of $\rho$. Because of the less computational burden in calculation of $\rho$, [see (5), (6)], in this paper we concentrate on the power variance and assess its value for the random phase updating algorithm. However, using (7) the corresponding value of PAPR can also be obtained.

\section{RANDOM PHASE UPDATING AlgORITHM}

As shown in Fig. 1 in the random phase updating algorithm for each carrier a random phase is generated and assigned to that carrier. Using (2) the OFDM signal with phasing is written as

$$
s(t)=\sum_{m=0}^{M-1} b_{m} e^{j 2 \pi\left((m / T) t+\phi_{m}\right)}
$$

where $2 \pi \phi_{m}$ is the $m$ th subcarrier phase shift. Adding random phases to each suibcarrier will change the power variance of OFDM signal. In the random phase updating algorithm, the phase of each subcarrier is updated by a random increment as:

$$
\left(\phi_{m}\right)_{i}=\left(\phi_{m}\right)_{i-1}+\left(\Delta \phi_{m}\right)_{i} \quad m=0,1, \ldots, M-1
$$

where $i$ is the iteration index and $\left(\Delta \phi_{m}\right)_{i}$ is the phase increment of the $m$ th subcarrier at $i$ th iteration. In the random phase updating method, without loss of generality, the initial phase, i.e., $\left(\phi_{m}\right)_{0}$, can be considered zero. Consequently, a random phase increment is generated and the phase is updated by adding the increment to the phase of that subcarrier. Flow chart of the algorithm for this iterative phase updating is shown in Fig. 2. In Fig. 2(A) a certain threshold for PV is set and for Fig. 2(B) a limited number of iterations is allowed.

In this paper different distributions for the random phase increments have been considered and their influence on the PV has been investigated. Two distributions are Gaussian $\left(\Delta \phi_{m}=\right.$ $\left.N\left(0, x^{2}\right)\right)$ and Uniform $\left(\Delta \phi_{m}=\right.$ Unif $\left.[0, x]\right)$, where $x \in$ $\{0.1,0.25,0.5,0.75,1\}$. Results are shown in Table I. It is seen that there is no significant difference in the PV results when Gaussian or Uniform distribution is considered for the phase increments. In the rest of the paper the uniform distribution has been chosen for the distribution of phase increments. The influence of different variances of the phase increments on the PV reduction of OFDM signal has been investigated. Results indicate a connection between phase shift variance and the number of iterations required reaching the threshold. Simulations have been carried out for different number of carriers as well as different PV thresholds. As shown in Fig. 3 when variance of phase shift increments is small more number of iterations is required. This can be clearly justified. When standard deviation of phase increments is small the generated phases are likely not good to reduce the PAPR. But when the standard deviation of phase increments is large, the random phase increments have larger variations and it is more likely that their values be proper to decrease the PV. As seen in Fig. 4 by increasing the standard deviation of phase increments the number of iterations to reach the threshold decreases. Meanwhile, the lower the PV threshold the more the number of iterations. That is quite clear since lower threshold or smaller PV needs more iterations to select the proper phases for the subcarriers. From Fig. 3 the influence of different number of carriers on the number of iterations for different variances of phase shifts is also clear. It is obvious that increasing number of

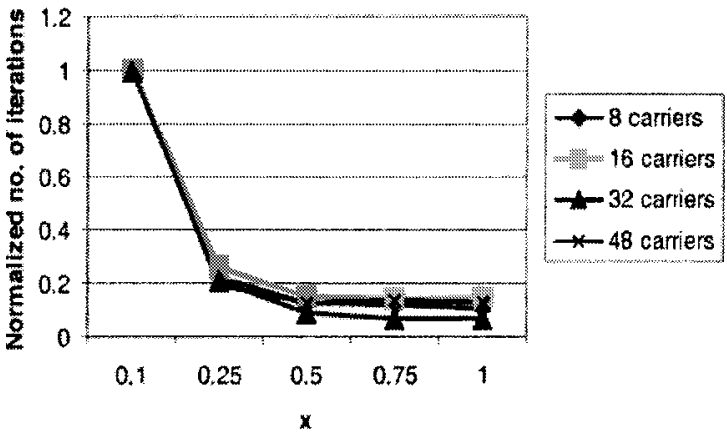

Fig. 3. Normalized mean number of iterations versus phase shift variance parameters $x$ for $M=\{8,16,32,48\}$ BPSK OFDM signal simulated with random phase updating algorithm (Fig. 2(A)).

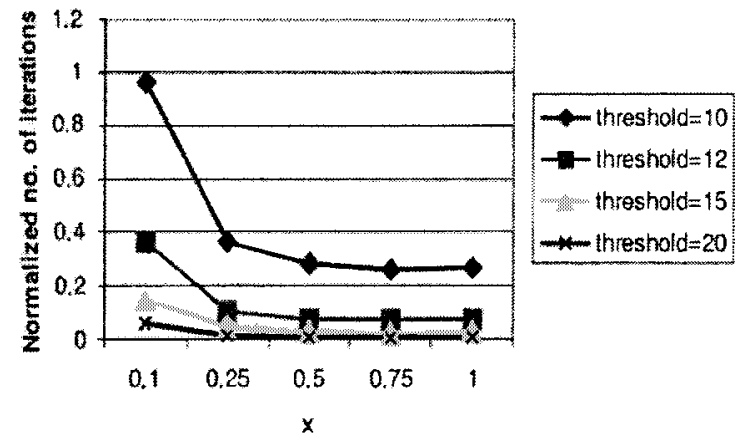

Fig. 4. Normalized mean number of iterations versus phase shift variance parameter $x$ for 8-carrier OFDM system and different threshold levels, simulated with random phase updating algorithm of Fig. 2(A)

carriers from 8 to 48 slightly changes the number of iterations of the algorithm. As shown in Fig. 4, and unlike the number of carriers, the threshold level has a significant effect on the number of iterations of the algorithm. Efficiency of the algorithm is mainly related to the selected threshold level and consequently number of iterations and not the number of carriers. This is why in Section IV the dynamic reduction of threshold is proposed.

Reducing of the PAPR with phasing implies a high degree of complexity and side information. For large number of carriers the computational burden for the calculation of $\mathrm{PV}$ is increased [see (5)]. Besides, because of more carriers more phases are involved in the algorithm which leads to more side information. The phase shifts have to be known at the transmitter and receiver. To lessen the problem, the quantization and grouping of the random phase increments has been carried out. Quantization of the phase shift to BPSK (i.e., 2) or QPSK (i.e., 4) type phase shifts (i.e., $\Delta \phi_{m} \in\{0,0.5\}$ or $\{0,0.25,0.5,0.75\}$, respectively) decreases the number of bits necessary to represent each phase shift which leads to a reduced complexity of the algorithm. Grouping means subcarriers are bundled and all subcarriers in the same bundle (group) get the same phase shift increment (see Fig. 5). By grouping the complexity of the algorithm is further reduced. Simulations were carried out for a 16-carrier OFDM for two and four levels of phase quantization and different number of iterations (Fig. 6). Results shown in Fig. 6 indicate that rounding of the phase increments to two levels does not change the variance and reduces the mean of PV. Grouping for 16 carrier BPSK-OFDM was examined with 2 groups of 8 carriers, 4 groups of 4 carriers and 8 groups of 2 carriers and 


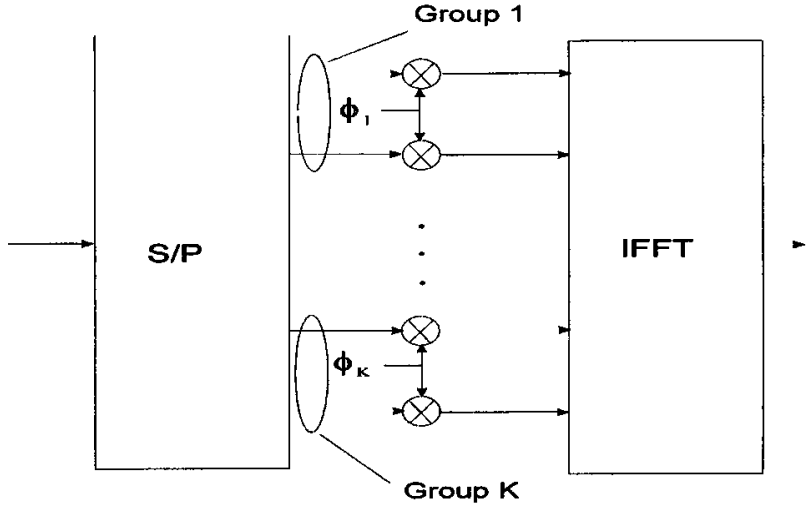

Fig. 5. Block diagram illustrating the grouping of the phases.

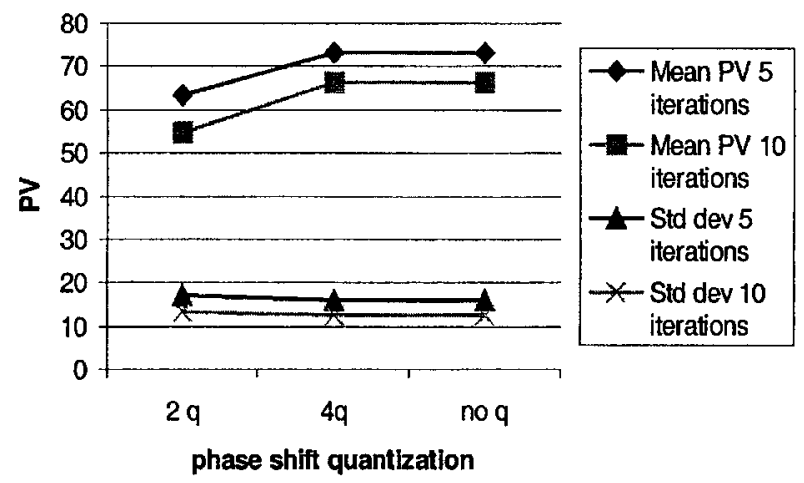

Fig. 6. Mean and standard deviation of power variance versus the phase quantization level for different number of iterations. OFDM with 16 carriers and BPSK modulation.

for different number of iterations. Parts of results are shown in Figs. 7 and 8. Results depict that 2 groups of each 8 carriers increase the PV when compared with 4 groups of 4 carriers and 8 groups of each 2 carriers. It should be mentioned that for further complexity reduction of the phasing algorithm, combination of grouping and quantization can also be applied.

In order to have an estimate of the amount of side information required with this algorithm let us consider a 16-carrier OFDM with QPSK modulation and without quantization and grouping of the phases. For representing the phase information of 16 carriers 4 bits are required. That means the amount of side information will be $4 / 32=12.5 \%$. By grouping of the carriers (e.g., into four groups) and without quantization, the amount of side information will be $2 / 32=6.25 \%$, and if a 4-level quantization is used, the amount of side information will reduce to $2 /(4 \times 32)=$ $1.56 \%$.

\section{Phase Updating With DyNAMIC Threshold}

In the random phase increment algorithm until now the PV threshold (used for comparison) was fixed (see Fig. 2). Following the selection of random phase increments it is possible to reduce the PV threshold. As illustrated in Fig. 9, by this approach the threshold level of the algorithm is dynamically reduced. When compared to the case of fixed threshold, this algorithm provides a lower PAPR. The first step of the algorithm is to calculate the PV of the original OFDM symbol, and then set the first threshold to, e.g., 10\% lower. Then starting from zero initial phases, the random phase shifts are generated
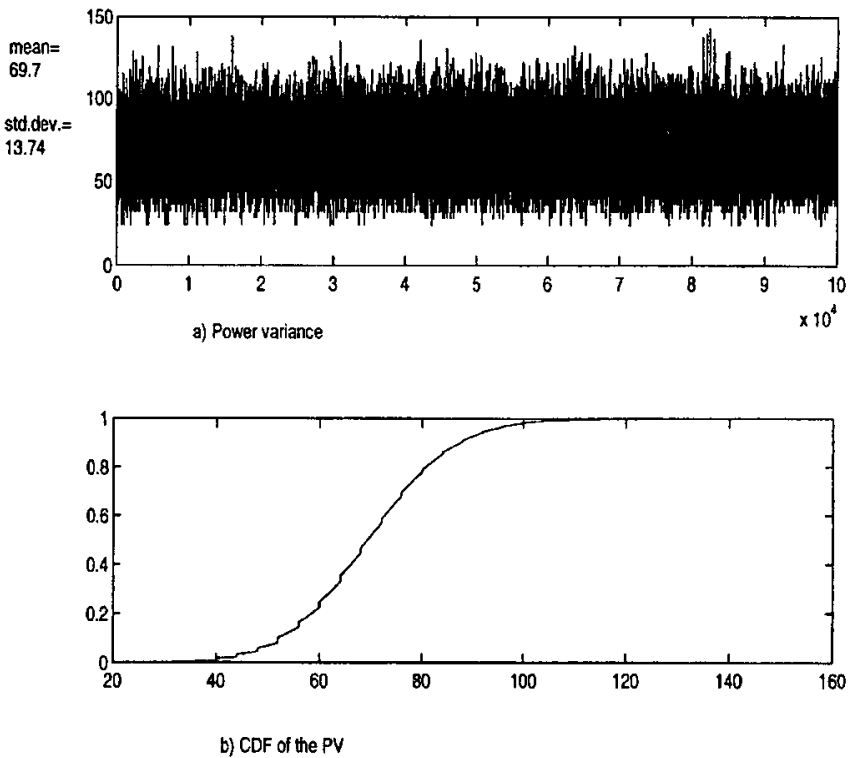

Fig. 7. Grouping of 16 carrier OFDM to 8 groups of 2 carriers. (a) Power variance versus symbol no., (b) $\mathrm{CDF}$ of the power variance.
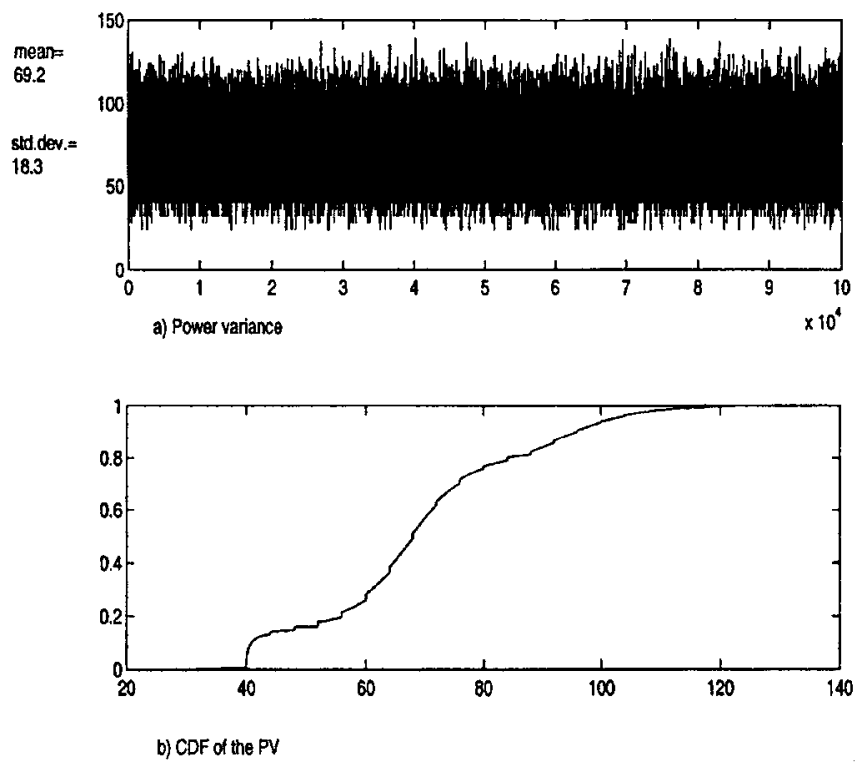

Fig. 8. Grouping of 16 carrier OFDM to 4 groups of 4 carriers. (a) Power variance versus symbol no., (b) $\mathrm{CDF}$ of the power variance.

and added to the symbols [see (9)]. Then with these phases the PV is calculated and compared with the threshold. If the PV is below the threshold, the phase increments are good and phases are updated and subsequently a new (reduced) threshold is made. Otherwise the new phase increments are generated and combined with the symbols, and the threshold is not changed. When the algorithm has made the new phase increments for a specific number of times subsequently without updating, the variance of the phase shift is changed. The algorithm stops when the variance of phase shift increments has reached a certain value. The algorithm was investigated for different sizes of threshold change, different number of iterations and different sizes of phase variance change and compared with the OFDM without this algorithm. Results are shown in Fig. 10. The description of the parameters in the horizontal axis of this figure 


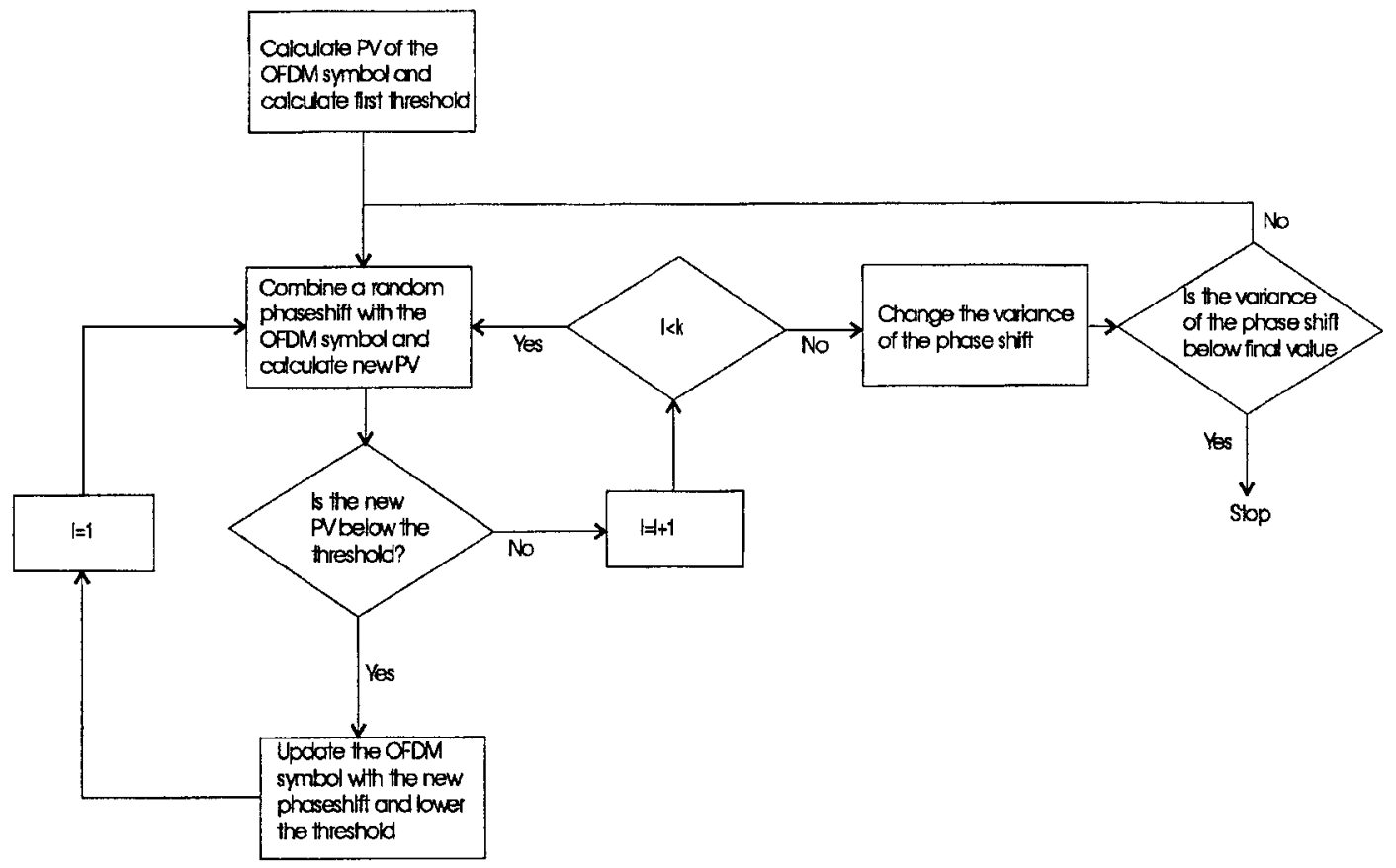

Fig. 9. Flowchart of the random phase updating algorithm with dynamic threshold.

is as follows: For A1 and A2 Threshold change $=10 \%$, Phase variance change $20 \%$ but Final phase variance for $\mathrm{A} 1$ is 0.1 and for A2 is 0.01 . For B1 and B2 Threshold change $=50 \%$, Phase variance change 20\% but Final phase variance for B1 is 0.1 and for $\mathrm{B} 2$ is 0.01 . For $\mathrm{C} 1$ and $\mathrm{C} 2$ Threshold change $=10 \%$, Phase variance change $10 \%$ but Final phase variance for $\mathrm{C} 1$ is 0.1 and for $\mathrm{C} 2$ is 0.01 . For D1 and D2 Threshold change $=50 \%$, Phase variance change 10\% but Final phase variance for D1 is 0.1 and for D2 is 0.01 . The number of iterations indicated in the viewgraph denotes the number of iterations between phase increments variance changes, i.e., if five successive iterations did not reduce the PV below the threshold, then the variance of the phase shift is reduced according to what described above. Fig. 10 shows the simulation results for the 16-carrier BPSK OFDM symbols. Results of dynamic threshold phase updating algorithm show that PV mean and variance reduce with more iterations. Meanwhile, for the allowed number of iterations, the mean of PV slightly changes with the threshold and phase variance change. While the variance of PV reduces faster with threshold and phase variance change.

To show how much PV using the random phase increment algorithm with dynamic threshold, is reduced simulations were run without the algorithm and one with the dynamic threshold and 100 iterations between reducing of the phase shift variance and parameter D1 explained earlier. The simulations were carried out for both 8 and 16 carrier BPSK OFDM symbols. For an 8-carrier OFDM the mean PV (or according to (7) and assuming $\beta=0.99$, the PAPR) for the simulation without random phase updating was 28.91 (and for mean of PAPR was 0.1373) with a standard deviation (Std) of PV equal to 20.59 (and Std of PAPR $=0.1162$ ), and the maximum PV equal to 140 (maximum PAPR as 0.2969). For the random phase updating with dynamic threshold algorithm mean PV was 5.71 (mean PAPR = 0.0652), Std of PV equal to 0.877 (Std of PAPR $=0.046)$ and a maximum value $\mathrm{PV}$ as 8.0 ( $\mathrm{max}$ of PAPR $=0.0751)$. This gives

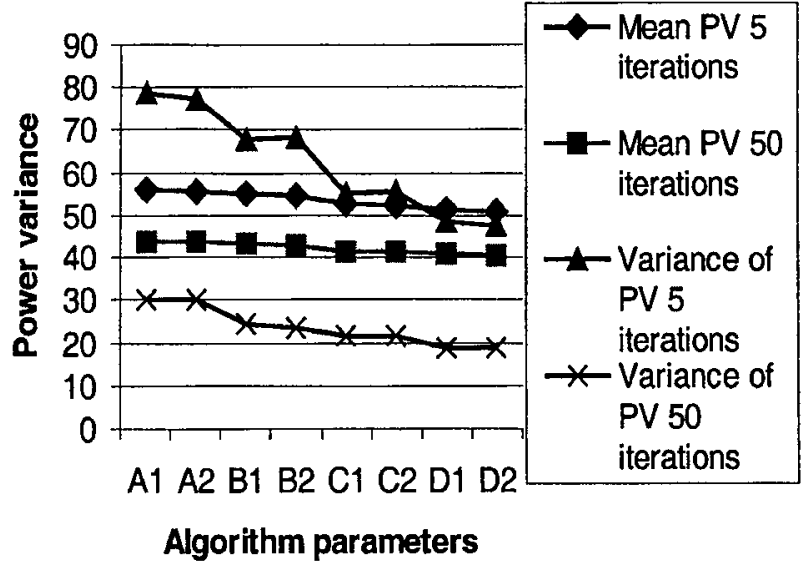

Fig. 10. Mean of PV for 16-carrier OFDM with random phase updating algorithm and dynamic threshold.

a $7 \mathrm{~dB}$ reduction of mean $\mathrm{PV}$ (or $3.23 \mathrm{~dB}$ reduction of mean PAPR), $13.7 \mathrm{~dB}$ reduction of Std of PV (or $4 \mathrm{~dB}$ reduction of Std of PAPR) and a $12.4 \mathrm{~dB}$ reduction of maximum PV (or $6 \mathrm{~dB}$ reduction of maximum PAPR). The simulations for the 16-carrier OFDM system introduced a $5 \mathrm{~dB}$ reduction of the mean $\mathrm{PV}$ ( $2.5 \mathrm{~dB}$ reduction of mean PAPR) and a $14.1 \mathrm{~dB}$ reduction of maximum PV (or around $7 \mathrm{~dB}$ reduction of max of PAPR) for the dynamic threshold algorithm.

\section{Bit ERror Rate Performance}

In the previous sections we have seen that proper phase updating of the OFDM signal reduces the PAPR. It is worth mentioning that because of phasing, the proposed algorithm does not impact the side lobes in the spectrum of the OFDM signal. It should be also emphasized that changing the phase of OFDM signal does not change the BER performance of the system if 


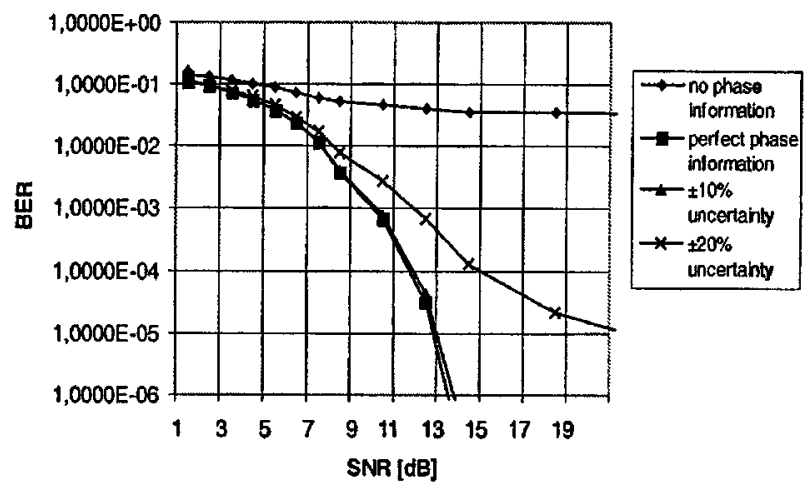

Fig. 11. BER performance versus signal-to-noise ratio for a 16-carrier OFDM system with different phase information errors at the receiver.

the phase changes are completely known to the receiver. This however implies a large amount of side information, especially if quantization and grouping are not applied. Fig. 11 illustrates how the BER performance of OFDM system with imperfect knowledge of the added phases is degraded. It is seen that up to $10 \%$ uncertainty (error) in the phase information at the receiver, (as a result of phase updating algorithm), slightly degrades the BER performance of the system.

\section{CONCLUSION}

In this paper the novel random phase updating algorithm was proposed to lessen the PAPR of the OFDM signal. In this algorithm phase of each subcarrier is updated by a random increment and the process is iterated until PAPR goes below a threshold level. The influence of different distributions for the phase shift increments and their variances on the mean power variance and number of iterations has been investigated. For reducing the complexity of the random phase updating algorithm, the quantization and grouping of the phase shifts was also carried out. Results showed that grouping and quantization of phases can reduce complexity without significant increase of the PAPR. To further decrease the PAPR, the random phase updating algorithm was extended with the dynamic threshold. In this case after successful updating of the phases the threshold level of the comparison is reduced and the variance of the phase increments is changed. Results show that for an 8-carrier OFDM system the random phase updating method with dynamic threshold reduces the mean power variance by a factor of $7 \mathrm{~dB}$ (or mean of PAPR by $3.23 \mathrm{~dB}$ ). Further in the paper, the effect of the imperfect knowledge of the phases (used for PAPR reduction) at the receiver, on the BER performance of the system has been investigated.

\section{REFERENCES}

[1] T. A. Wilkinson et al., "Block coding scheme for reduction of peak to mean envelope power ratio of multicarrier transmission schemes," Electron. Lett., vol. 30, no. 25, 1994.
[2] R. F. H. Fischer et al., "Reducing the peak-to-average power ratio of multicarrier modulation by selected mapping," Electron. Lett., vol. 32, no. 22, 1996.

[3] G. Wade et al., "Peak-to-average power reduction for OFDM schemes by selective scrambling," Electron. Lett., vol. 32, no. 21, 1996.

[4] S. H. Muller and J. B. Huber, "OFDM with reduced peak-to-average power ratio by optimum combination of partial transmit sequences," Electronics Letters, vol. 33, no. 5, 1997.

[5] M. Friese, "Multitone signals with low crest factor," IEEE Trans. Commun., vol. 45, Oct. 1997.

[6] J. Tollado, Multicarrier Modulation With Low PAR, MA: Kluwer Publishers, 2000.

[7] G. L. Stuber and D. Kim, "Clipping noise mitigation for OFDM by decision aided reconstruction," IEEE Commun. Lett., vol. 3, 1999.

[8] R. J. van Nee and A. de Wild, "Reducing peak-to-average power ratio of OFDM," in IEEE Vehic. Technol. Conf., 1996, pp. 2072-2076.

[9] R. Prasad and R. J. van Nee, OFDM for Wireless Multimedia Communications. Boston, MA: Artech House, 1999.

[10] O. Muta et al., "Peak power suppression with parity carrier for multicarrier transmission," in IEEE Vehic. Technol. Conf. (VTC'99-Fall), 1999, pp. 2923-2928.

[11] H. Nikookar and R. Prasad, "Weighted multicarrier modulation for peak-to average power reduction," IEICE Trans. Commun., vol. E83-B, Aug. 2000.

[12] X. Wang and T. T. Tjhung, "Reduction of peak-to-average power ratio of OFDM system using companding technique," IEEE Trans. Broadcast., vol. 45, Sept. 1999

[13] Y. S. Chu et al., "On compensating nonlinear distortions of an OFDM system using efficient an adaptive predistorter," IEEE Trans. Commun., vol. 47, Apr. 1999.

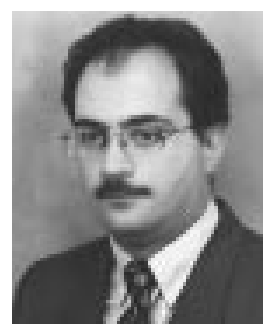

Homayoun Nikookar received his B.Sc. and M.Sc. (with distinction) in Electrical Engineering from Sharif University of Technology, Tehran, Iran, in 1986 and 1987, respectively; and his Ph.D. from Delft University of Technology (TUDelft), the Netherlands, in 1995. From 1988 to 1992 he served as a lecturer at the Department of Electrical Engineering of AmirKabir University of Technology (Tehran Polytechnic Institute), where he also carried out research on the characterization of indoor radio propagation channels. In 1993 he started as a guest researcher at the TUDelft. After receiving his Ph.D. in 1995 he got a postdoc research associate position at the International Research Center for Telecommunications-Transmission and Radar of the TUDelft, where in 1999 he was appointed as an assistant professor. He is co-recipient of the 2000 IEEE Neal Shepherd Best Propagation Paper Award for publication in the March 2000 issue of the Trans. on Vehicular Technology. He is also co-recipient of the Best Paper Award of the International Symposium on Personal Indoor Mobile Radio Communiactions, Toronto, 1995. His areas of interest include wireless channel modeling, multicarrier transmision, and CDMA.

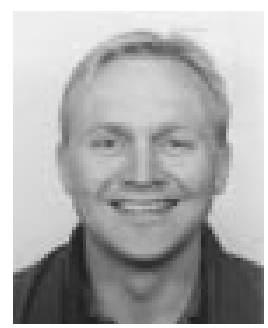

Knut Sverre Lidsheim received his B.Sc. in Microelectronics from Technical University of Kongsberg, Norway; and his M.Sc. from Delft University of Technology, the Netherlands, in 2001. $\mathrm{He}$ is currently working at Kongsberg Defense Communication in Oslo, developing a new handheld radio for tactical communications. 